\title{
Bilateral Dorsal Cochlear Nucleus Lesions Prevent Acoustic-Trauma Induced Tinnitus in an Animal Model
}

\author{
Thomas Jeffrey Brozoski ${ }^{1}$, Kurt W. Wisner ${ }^{1}$, Lauren T. Sybert ${ }^{1}$, and Carol A. Bauer ${ }^{1}$ \\ ${ }^{1}$ Division of Otolaryngology—Head and Neck Surgery, Southern Illinois University School of Medicine, Springfield, IL 62794, USA
}

Received: 18 October 2010; Accepted: 26 August 2011; Online publication: 4 October 2011

\begin{abstract}
Animal experiments suggest that chronic tinnitus ("ringing in the ears") may result from processes that overcompensate for lost afferent input. Abnormally elevated spontaneous neural activity has been found in the dorsal cochlear nucleus (DCN) of animals with psychophysical evidence of tinnitus. However, it has also been reported that DCN ablation fails to reduce established tinnitus. Since other auditory areas have been implicated in tinnitus, the role of the DCN is unresolved. The apparently conflicting electrophysiological and lesion data can be reconciled if the DCN serves as a necessary trigger zone rather than a chronic generator of tinnitus. The present experiment used lesion procedures identical to those that failed to decrease pre-existing tinnitus. The exception was that lesions were done prior to tinnitus induction. Young adult rats were trained and tested using a psychophysical procedure shown to detect tinnitus. Tinnitus was induced by a single unilateral high-level noise exposure. Consistent with the trigger hypothesis, bilateral dorsal DCN lesions made before highlevel noise exposure prevented the development of tinnitus. A protective effect stemming from disruption of the afferent pathway could not explain the outcome because unilateral lesions ipsilateral to the noise exposure did not prevent tinnitus and unilateral lesions contralateral to the noise exposure actually exacerbated the tinnitus. The DCN trigger mechanism may involve plastic circuits that, through loss of inhibition, or upregulation of excitation, increase spontaneous neural output to rostral areas such as the inferior colliculus. The
\end{abstract}

Correspondence to: Thomas Jeffrey Brozoski · Division of Otolaryngology-Head and Neck Surgery - Southern Illinois University School of Medicine · Springfield, IL 62794, USA. Telephone: +1-217-545-6583; fax: +1-217-545-6583; email: tbrozoski@siumed.edu increased drive could produce persistent pathological changes in the rostral areas, such as high-frequency bursting and decreased interspike variance, that comprise the chronic tinnitus signal.

Keywords: auditory brainstem, hearing disorder, trigger zone, prevention

\section{INTRODUCTION}

Animal models have contributed to the emergent neuroscience of tinnitus (Bauer and Brozoski 2001; Brozoski and Bauer 2008; Guitton et al. 2003; Heffner and Harrington 2002; Jastreboff et al. 1988; Kaltenbach and Heffner 1999; Lobarinas et al. 2004; Ruttiger et al. 2003). A working hypothesis is that hearing loss, which often may be subclinical, leads to a downregulation of inhibition and to reorganization of the central auditory system (Brozoski et al. 2007b; Eggermont and Roberts 2004). Downregulation of inhibition may be a homeostatic compensation for decreased input. Overcompensation may produce the sensation of sound without stimulation, i.e., tinnitus.

The dorsal cochlear nucleus (DCN) has been identified as a contributor to tinnitus pathology (Kaltenbach 2006). Chronically elevated neural activity has been found in the DCN days (Kaltenbach and Afman 2000) to months after high-level sound exposure (Brozoski et al. 2002). Several features of the DCN may contribute to its role in tinnitus. Sound localization and signal processing in poor acoustic environments (Nelken and Young 1996; Oertel and Young 2004; Reiss et al. 2007) involve adaptive signal processing. Plastic mechanisms in the DCN necessary for adaptive signal processing may provide a substrate 
for overcompensation after peripheral trauma. The cerebellar-like circuitry of the DCN suggests that inhibition is an important component of that processing. Cerebellar circuits are characterized by their interconnected inhibitory control loops (Ito 1984). Direct application of GABA agonists and antagonists to the DCN dramatically influences the response characteristics of single units (Backoff et al. 1999; Caspary et al. 1987). Pathological upregulation of somatosensory input to the DCN following trauma also has been hypothesized to contribute to tinnitus (Dehmel et al. 2008; Shore et al. 2008). Downregulation of inhibition, potentially in concert with upregulation of somatosensory input, could explain DCN hyperactivity and tinnitus.

If the DCN is a tinnitus signal source, ablating the DCN in animals with tinnitus should decrease their tinnitus. Using an established animal model, rats with psychophysical evidence of tinnitus had focal lesions made in the dorsal DCN several months after tinnitus induction (Brozoski and Bauer 2005). The lesions interrupted rostral DCN output, but they did not decrease the established tinnitus (Brozoski and Bauer 2005). Failure of DCN lesions to decrease tinnitus suggests that the DCN is not a necessary source of the chronic signal. Alternatively, the DCN may serve as a trigger zone. Hyperactivity in the DCN following acoustic insult has been reported to occur within days of high-level sound exposure (Kaltenbach et al. 2000).

In the present study, the DCN was lesioned prior to traumatic sound exposure. This was done to test the hypothesis that the DCN serves as a necessary trigger zone for the pathological cascade leading to tinnitus. Surgical, psychophysical, and histological methods were identical to those used in the previous study showing no reduction in tinnitus following postexposure ablation (Brozoski and Bauer 2005). If the DCN is an obligatory trigger zone for tinnitus pathology, pre-exposure ablation of the DCN should reduce or eliminate the acoustic induction of tinnitus.

\section{METHOD}

The methods used in the present experiment were identical to those reported in Brozoski and Bauer (2005). The exception was that DCN lesioning was done before the induction of tinnitus.

\section{Subjects}

Forty-eight adult male Long-Evans rats (Harlan, Indianapolis, IN, USA), 3 months old at the start of the experiment, were individually housed and main- tained at $25^{\circ} \mathrm{C}$ with a $12 / 12$-h reversed light/dark schedule. Two rats were eliminated for health reasons early in the experiment.

\section{Behavior: initial training}

Prior to surgical intervention, the rats were placed on restricted food intake and trained to lever press for food pellets (45 mg, MLab Rodent, TestDiet, Richmond, IN, USA). Diet restriction was individually tailored to each subject, sufficient to produce a minimum of 200 lever presses per session when fully trained, with less than $10 \%$ within-session variation (session average lever presses for all rats-1,009, \pm 346 $\mathrm{SD})$. Supplemental food was given at the conclusion of each session sufficient to maintain body weight at or above $80 \%$ of normative age-weight values. Five $60-\mathrm{min}$ sessions were run per week. Training and testing occurred in individual commercial operant conditioning chambers (Lafayette Instruments, Mod. 80001, Lafayette, IN, USA). Training required approximately 4 weeks to achieve criterion-level performance on a 20-s variable-interval reinforcement schedule (on average, a food pellet was available $20 \mathrm{~s}$ after the previous pellet). Broadband noise (BBN) was presented to each operant chamber via a speaker (Optimus, 40-1219, Tandy), center mounted in each chamber lid (calibration procedure described below). In training, BBN was constantly present at $60 \mathrm{~dB}$ sound pressure level (SPL). Experimental control and data acquisition were accomplished using desktop computers running in-house programs and custom interfaces (Keithley/MetraByte, Cleveland, OH, USA). At the conclusion of initial training, the animals were given DCN dorsal lesions.

\section{Lesions}

DCN lesions were done prior to traumatic sound exposure. Group composition as confirmed by histological analysis (described below) is summarized in Table 1. Subjects were deeply anesthetized with a ketamine $\mathrm{HCl}$ (50 mg/kg) and xylazine $(9 \mathrm{mg} / \mathrm{kg}$ ) mixture and placed in a stereotaxic head frame. The stereotaxic frame was equipped with blunt ear bars that protected the tympanic membrane. The scalp was reflected using a midline incision, and two 2-mm diameter craniotomies were made $3 \mathrm{~mm}$ posterior to lambda and $\pm 3 \mathrm{~mm}$ lateral of midline. A temperature sensing radio-frequency electrode (Radionics, Mod. TC, Burlington, MA, USA; $0.3 \mathrm{~mm}$ tip diameter) was lowered along a $4^{\circ}$ laterally angled track to reach the dorsal aspect of the DCN, $6.1 \mathrm{~mm}$ below the surface of the dura mater (Fig. 1). Radio-frequency current (Radionics, RF4G, Burlington, MA, USA) was applied for $80 \mathrm{~s}$ to the electrode, with current level adjusted to 
TABLE 1

\begin{tabular}{|c|c|c|c|c|}
\hline \multicolumn{5}{|c|}{ Group composition and subject number } \\
\hline Acoustic exposure & Bilateral & Ipsilateral & Contralateral & Bilateral inaccurate and sham-lesioned \\
\hline Exposed & 8 & 4 & 3 & 8 \\
\hline Unexposed & 6 & 3 & 4 & 10 \\
\hline
\end{tabular}

Acoustic exposure refers to the tinnitus induction procedure. DCN lesion designations, bilateral, ipsilateral, contralateral, and inaccurate, were based on histological criteria. Subjects with bilaterally inaccurate lesions were combined with subjects in the sham-lesion group. Data were combined because psychophysical performance was equivalent in the two groups

maintain a $70^{\circ} \mathrm{C}$ tip temperature. These parameters were sufficient to produce a 1-2-mm diameter lesion. After lesioning, the electrode was withdrawn and Gelfoam placed in the craniotomy. A contralateral lesion was made using the same procedure. Every animal in the ablated group was bilaterally targeted for DCN lesions. At the conclusion of surgery, the scalp was closed with wound clips and the animal given a $5 \%$ ibuprofen solution to drink for 3 days. A minimum of 10 days elapsed before behavioral training resumed. At the conclusion of psychophysical testing, lesion size and accuracy were assessed in histological sections, described below. On the basis of histological analysis, the animals were later classified as having bilateral lesions, unilateral (ipsilateral or contralateral to acoustic exposure) lesions, or control (insufficient or inaccurately placed) lesions, and their data analyzed accordingly. Final group composition is summarized in Table 1 . The procedure for sham-surgery animals was identical, except that electrodes were not lowered into the brain.

\section{Behavior: the suppression ratio, a running relative measure}

Behavioral training resumed after recovery from surgery. The behavior of interest was lever pressing

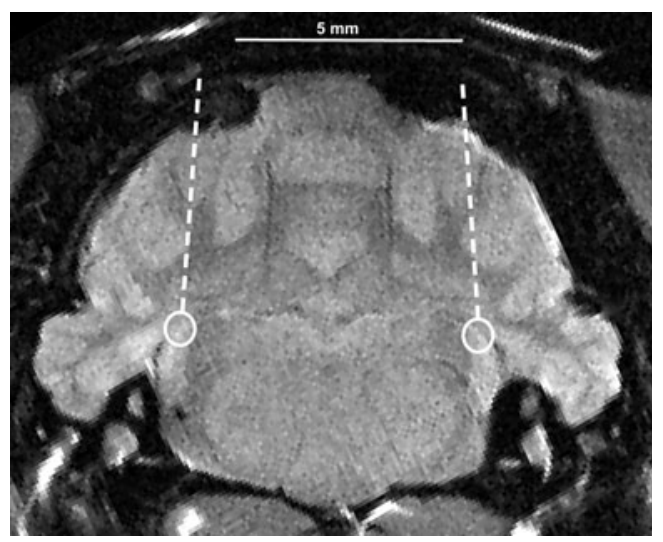

FIG. 1. Dorsal cochlear nucleus (DCN) ablation target area and electrode approach. Bilateral radio-frequency lesions $1-2 \mathrm{~mm}$ in diameter were targeted at the dorsal aspect of the DCN (circled), thereby interrupting its major rostral output. MRI transverse image from a rat of the same age and strain as used in the present experiment. Section plane was approximately $3 \mathrm{~mm}$ posterior to lambda. during randomly presented test sounds. Lever pressing was quantified using a relative rate measure, the suppression ratio $(R) . R$ was determined as a running measure for successive 1-min segments of each session using the formula $R=B /(A+B)$, where $A$ was the number of lever presses in the preceding 1-min segment and $B$ the number of lever presses in the current 1 -min segment. $R$ can vary between 0 and 1 . A value of 0 is attained when lever pressing in the current minute is 0 , a value of 0.5 when lever pressing in the current minute is equal to that of the previous minute, and a value of 1 when lever pressing in the previous minute is $0 . R$ provided a running index of behavior, in 1-min segments, and enabled a quantitative comparison between subjects as well as unbiased compilation of group data. $R$ is a useful index of perceptual performance in that it is very sensitive to short-term behavioral effects, such as those produced by sensory events, but it is very insensitive to gradual behavioral effects, such as those produced by changes in motivational status, for example, satiation.

\section{Behavior: acclimation to acoustic variation}

Following initial training, and after recovery from lesion surgery, acoustic test stimuli were introduced using a procedure designed to acclimate subjects to the presentation of stimuli other than BBN. During stimulus introduction, which occurred over six 1-h sessions, all behavioral contingencies remained the same as in training. Ten acoustic stimuli were digitally synthesized (Stanford Research Systems, DS-345, Palo Alto, CA, USA) and individually presented for $60 \mathrm{~s}$ over the lid-mounted speakers during the test session. Stimulus presentations could not occur within $2 \mathrm{~min}$ of one another, or within $2 \mathrm{~min}$ of the beginning or end of the session. Two of the ten presentations were always speaker-off periods. The remaining eight presentations were either $\mathrm{BBN}$, or 10,16 , or $20-\mathrm{kHz}$ tones presented at four different intensity levels, randomly ordered, with the sound levels extending across the subject's sensitivity range. Each test stimulus level was repeated once within the session. Test stimulus type, i.e., $\mathrm{BBN}, 10 \mathrm{kHz}$, etc., varied randomly between sessions, but remained constant within a 
session. Background sound (BBN, $60 \mathrm{~dB}$, SPL) was off during the test stimulus presentations and otherwise on.

\section{Acoustic exposure and sound calibration methods}

At the conclusion of acclimation, lesioned and shamlesioned subjects, as specified in Table 1, were exposed once to high-level sound. These subjects will be referred to as "exposed." Treated identically, but unexposed, were a comparable number of lesioned and sham-lesioned subjects (see Table 1). These subjects will be referred to as "unexposed." All subjects, exposed and unexposed, were anesthetized to an areflexive state with an isoflurane $/ \mathrm{O}_{2}$ mixture (Aerrane, Baxter Healthcare Corp., Deerfield, IL, USA) placed in a masked head holder, and had their hearing thresholds determined using auditory brainstem-evoked potentials (ABR, described below). The exposed subjects were then exposed once unilaterally for $60 \mathrm{~min}$ to band-limited noise (similar to Bauer and Brozoski 2001; Brozoski et al. 2007a, b). The exposure stimulus was produced using a noise generator (Grayson-Stadler 1724, Eden Prairie, MN 55344, USA), bandpass filter (KrohnHite 3384, 8 pole Butterworth filter, Brockton, MA, USA), and audio amplifier (55ES, Sony, New York, NY, USA), delivered monaurally using a speaker driver (FT17H, Fostex, Tokyo, Japan) in a custom enclosure funneling the sound to a flexible tube that fit into the auditory canal. Peak stimulus intensity, centered at $16 \mathrm{kHz}$, was $116 \mathrm{~dB}$ (SPL), with an approximately linear decay to ambient levels at 6 and $24 \mathrm{kHz}$. Acoustic values were calibrated using a Brüel \& Kjaer (Norcross, GA, USA) Pulse sound measurement system (Pulse 13 software), equipped with a 3560C high-frequency module, and a 4138 pressure-field microphone (Brüel \& Kjaer) coupled to the transducer using rubber tubing with the internal dimensions of an adult rat external auditory canal. The sound measurement system permitted linear sound intensity measurements between 0 and $140 \mathrm{~dB}$ (re $20 \mu \mathrm{Pa}$ ) and spectral analysis between $6.5 \mathrm{~Hz}$ and $100 \mathrm{kHz}$. Calibrations were carried out as unweighted linear SPLs. All sound levels reported in the present experiments are unweighted measures.

Sound levels were calibrated in the operant test chambers using the Brüel \& Kjaer Pulse system described above, equipped with a Brüel \& Kjaer 4191-L free-field microphone. This system permitted linear sound level measurements to be made between 0 and $140 \mathrm{~dB}$ (re $20 \mu \mathrm{Pa}$ ) with spectral resolution between $3.15 \mathrm{~Hz}$ and $40 \mathrm{kHz}$. The microphone was positioned in each test chamber at a location $10 \mathrm{~cm}$ below the lid-mounted speaker, in the approximate location of a rat's head during testing. A cloth bundle approximating the volume of a rat was placed in the test chamber along with the microphone to distort the sound field as it would be by a rat.

\section{Behavior: suppression training}

Suppression training followed stimulus acclimation. In suppression training, the subjects received a 1-s, $0.5-\mathrm{mA}$ foot shock through the grid floor of the test chamber if they lever-pressed above a criterion level in speaker-off periods. When scheduled, only one foot shock was given at the conclusion of the speaker-off period. The purpose of suppression training was twofold: It trained the subjects to listen carefully to their acoustic environment throughout the session because foot shocks could only be avoided if the unpredictable speaker-off periods were detected. Secondly, it trained subjects to discriminate between the speaker-on and speaker-off periods. A single foot shock was given at the end of a speaker-off period if $R \geq 0.1$. When subjects decreased their lever pressing during speakeroff periods, so that $R<0.1$, foot shock was avoided. Whenever the speaker was on, irrespective of the acoustic signal, lever presses never led to foot shock. The foot shock contingency during speaker-off periods remained in place throughout the experiment, including the acoustic test sessions described below. As such, once suppression training was implemented, it remained a constant feature of the experimental procedure. On average, subjects received less than one foot shock per month for the remainder of the study. The tandem constant parameters of food reinforcement for lever pressing, and foot shock for speaker-off lever pressing, maintained discrimination behavior in a steady state.

\section{Behavior: post-exposure tinnitus testing}

Four different test stimuli were used to determine post-exposure tinnitus: BBN and 10-, 16-, and 20-kHz tones. One of the four stimuli was randomly selected and presented eight times within a session, using four different levels. Each level was repeated, for a total of eight $(4 \times 2)$ presentations per session. Every test series included two additional, randomly inserted, speakeroff periods. As in acclimation, test presentations and speaker-off periods were $60 \mathrm{~s}$ in duration, separated from one another, and the session start and end, by a minimum of $2 \mathrm{~min}$ of background sound. The acoustic stimuli were presented in pseudorandomly scheduled, 60-s test periods. Sound presentations were synchronized and identical in all chambers. All subjects were tested daily.

Exposed and unexposed subjects were treated identically and tested in parallel. Individual subject and group discrimination functions were derived from test- 
stimulus suppression-ratio data from the final 16 sessions where performance stability was maximal (4 sessions each for BBN, 10, 16, and $20 \mathrm{kHz}$ ).

Evidence of tinnitus was determined by the divergence of group discrimination functions. For subjects with tinnitus, test stimuli that resembled their tinnitus served as a signal for response (lever press) suppression. In contrast, for control subjects without tinnitus, the signal for suppression was silence. Test stimuli with sensory features resembling tinnitus should therefore produce greater suppression (i.e., fewer lever presses) in subjects with tinnitus. Previous research (Bauer and Brozoski 2001) has shown that Long-Evans adult rats unilaterally exposed to highlevel band-limited noise show evidence of tinnitus in a range between 10 and $30 \mathrm{kHz}$.

\section{Hearing thresholds}

ABR thresholds were obtained before and immediately after high-level sound exposure (if exposed), and at the conclusion of behavioral testing. ABR measurements were obtained using either a Tucker Davis Technologies System 3 Real Time Signal Processing System running BioSig32 and SigGen (Tucker Davis Technologies, Alachua, FL, USA), or an IHS Smart EP System, running IHS High Frequency Software (v. 2.33) and using IHS high-frequency transducers (HFT9911-20-0035, Intelligent Hearing Systems, Miami, FL, USA). Evoked responses were differentially recorded from a subcutaneous vertex needle electrode referenced to an electrode at the occiput. Evoked responses for 10-ms epochs following stimulus onset were amplified $100,000 \times$, bandpassfiltered $(100-3,000 \mathrm{~Hz})$, and averaged for 512 repetitions of each frequency-intensity level combination. Digitized records of the evoked responses (40- $\mu \mathrm{s}$ resolution) were exported to Excel (Microsoft, Redmond, WA, USA) for analysis and threshold determination. Hearing thresholds for each ear were defined by the lowest stimulus level that produced statistically distinct and visually distinct evoked waveforms, defined as the maximum peak-to-peak deflection within the $10-\mathrm{ms}$ window following stimulus onset. Statistical routines were custom applications written for Excel.

\section{Data analysis}

All data were entered into spreadsheets. For inclusion in final statistical analysis, individual subject psychophysical data sets had to pass two quality filters: (a) There had to be a minimum of 200 total lever presses in the session, and (b) the average suppression ratio during nontest periods (i.e., during background $(\mathrm{BBN})$ ) had to be at least 0.4. Descriptive and inferential statistical analyses and graphic depictions were done using Excel (Professional Edition, 2007, Microsoft, Redmond, WA, USA). Treatment groups were constituted on the basis of traumatic sound exposure, i.e., exposed vs. unexposed, and on the basis of surgical treatment as ultimately defined by histological analysis (procedure described below). There was no difference in psychometric performance between the inaccurate lesion and shamlesioned subjects, so their data were combined into a single group for analysis, hereafter referred to as "shamlesioned." Two-factor mixed ANOVAs were used to compare performance between the eight treatment groups (Table 1) within each stimulus condition (BBN, $10 \mathrm{kHz}$, etc.), with stimulus level as the repeated measure. The experimental protocol was approved by the Laboratory Animal Care and Use Committee of Southern Illinois University School of Medicine.

\section{Histology}

At the conclusion of psychophysical testing, and after final ABR assessment, subjects were given a lethal intraperitoneal dose of a commercial euthanasia agent (Sleepaway $^{\circledR}$, Fort Dodge Animal Health, Fort Dodge, IA, USA) and perfused transcardially with $0.9 \%$ normal saline followed by $4 \%$ paraformaldehyde in $0.1-\mathrm{M}$ phosphate buffer. The brain was extracted and stored in $4 \%$ paraformaldehyde at $2^{\circ} \mathrm{C}$. Prior to cryostat sectioning, the brains were immersed in $20 \%$ sucrose for $24 \mathrm{~h}$. Coronal sections, $40 \mu \mathrm{m}$ thick, encompassing the DCN, were obtained. Sections were mounted on glass slides, stained with fast H\&E (Hematoxylin 7211, Eosin-Y711, Richard-Allan Scientific, Kalamazoo, MI, USA), and digitally photographed using light microscopy. Lesions were graded independently by three experimenters for accuracy and sufficiency. Visual inspection and scoring took into account lesion placement and volume. Scoring was done without knowledge of the subject's treatment history (i.e., exposure, psychophysical performance, etc.). Lesions centered on the dorsal DCN and encompassing at least $50 \%$ of the medial-to-dorsal DCN were graded as sufficient (Fig. 2). Lesions encompassing less than $50 \%$ of the DCN, including lesions in nonauditory structures, were graded as insufficient. The histological outcome was used to reconstitute group membership before final statistical analysis.

\section{RESULTS}

\section{Lesions and group composition}

The ablation target and electrode approach are shown in Figure 1. Micrographs of representative bilateral and unilateral lesions are shown in Figure 2. 
A. Bilateral Lesion

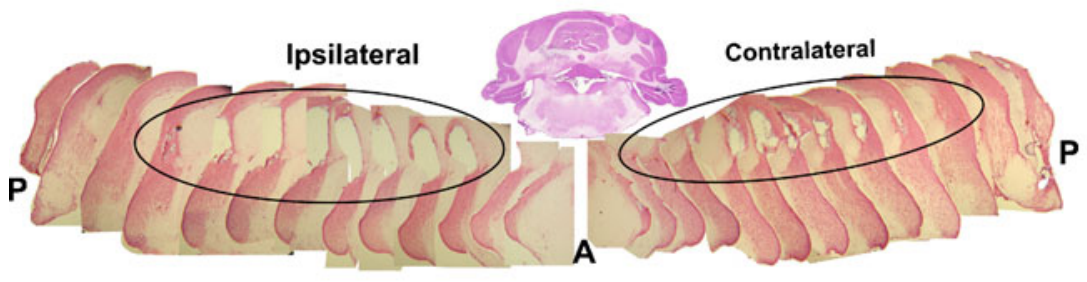

B. Ipsilateral Lesion

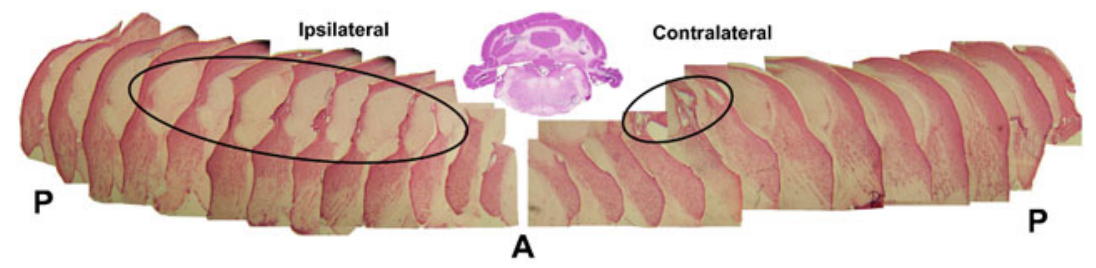

C. Contralateral Lesion

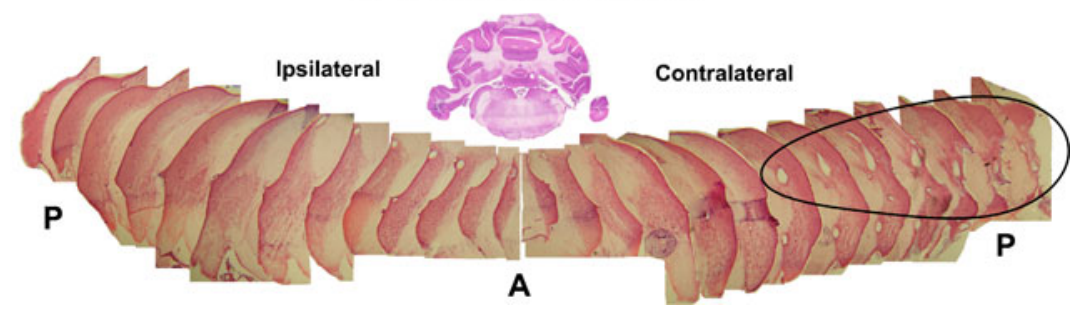

FIG. 2. Representative accurate and sufficient DCN lesions. Forty-micrometer frozen sections were made through the entire cochlear nucleus. Sections were stained with fast $\mathrm{H} \& \mathrm{E}$ as described. Serially reconstructed lesions are shown at 120-200 $\mu \mathrm{M}$ intervals, organized from anterior $(A)$ to posterior $(P)$ position. A Bilaterally sufficient lesions. Discernable tissue destruction was evident within the encircled range of sections. Most tissue destruction in this animal was evident in rostral to mid-caudal locations. B An ipsilaterally sufficient lesion. The small contralateral anterior lesion in this animal did not meet sufficiency criteria. C A contralaterally sufficient lesion, evident in the encircled range of section, with most tissue destruction in the caudal DCN.
In general, lesion sites were easy to recognize. Although the shape, volume, and location of the lesions varied, their perimeter had a characteristic fulminate appearance that was very different than that of other voids, such as those characteristic of ventricles and blood vessels. The lesions were photographed and quantified in all brain sections in which they appeared. Final group composition, summarized in Table 1, was determined by the histological criteria previously described. Psychophysical data from the inaccurate and insufficient lesion group were combined with data from the sham-lesioned group, since they did not significantly differ.

\section{Tinnitus: sham and inaccurate lesions}

In previous research, using similar sound exposure parameters, tinnitus was found to occur between 16 and $24 \mathrm{kHz}$, with the greatest effect at $20 \mathrm{kHz}$ (Bauer et al. 1999; Bauer and Brozoski 2001; Brozoski and Bauer 2005). These results were replicated in the present study, where significant evidence of tinnitus appeared at $20 \mathrm{kHz}$ in the exposed DCN-intact animals. The exposed animals showed a frequency-specific downshift in their discrimination functions, compared to unexposed controls (Fig. 3D). The downshift occurred because the animals were conditioned to suppress lever pressing during speaker-off periods, and speaker-off periods sounded different to exposed animals, compared to unexposed. Exposed animals should hear their tinnitus during speaker-off periods; they would then be expected to suppress lever pressing when tested with stimuli resembling their tinnitus. Between-group statistical comparisons are summarized in each panel of Figure 3 (mixed ANOVA, for stimulus levels above "speaker off").

\section{Tinnitus protection: bilateral lesions}

If, as hypothesized, the DCN serves as a necessary trigger zone, rats with sufficient pre-exposure DCN lesions should not develop tinnitus, while those with intact DCNs should. This result was obtained with criterion-level bilateral DCN lesions. Exposed rats with bilateral lesions did not display a tinnitus downshift (Fig. 4, square data points) and were significantly different than exposed DCN-intact rats (circular data points) for all test conditions (mixed ANOVA between-group statistics summarized each panel of Fig. 4). In contrast, exposed bilateral lesioned animals were not significantly different than unexposed bilateral lesioned animals (Fig. 4, triangular data points; $F_{1,64}=0.13$ to $3.42 ; p=0.720$ to 0.069$)$. The two-way comparison between exposed bilateral lesioned animals, unexposed bilateral lesion animals, and exposed DCN-intact animals indicates that bilateral lesioned animals were not psychophysically affected by the 

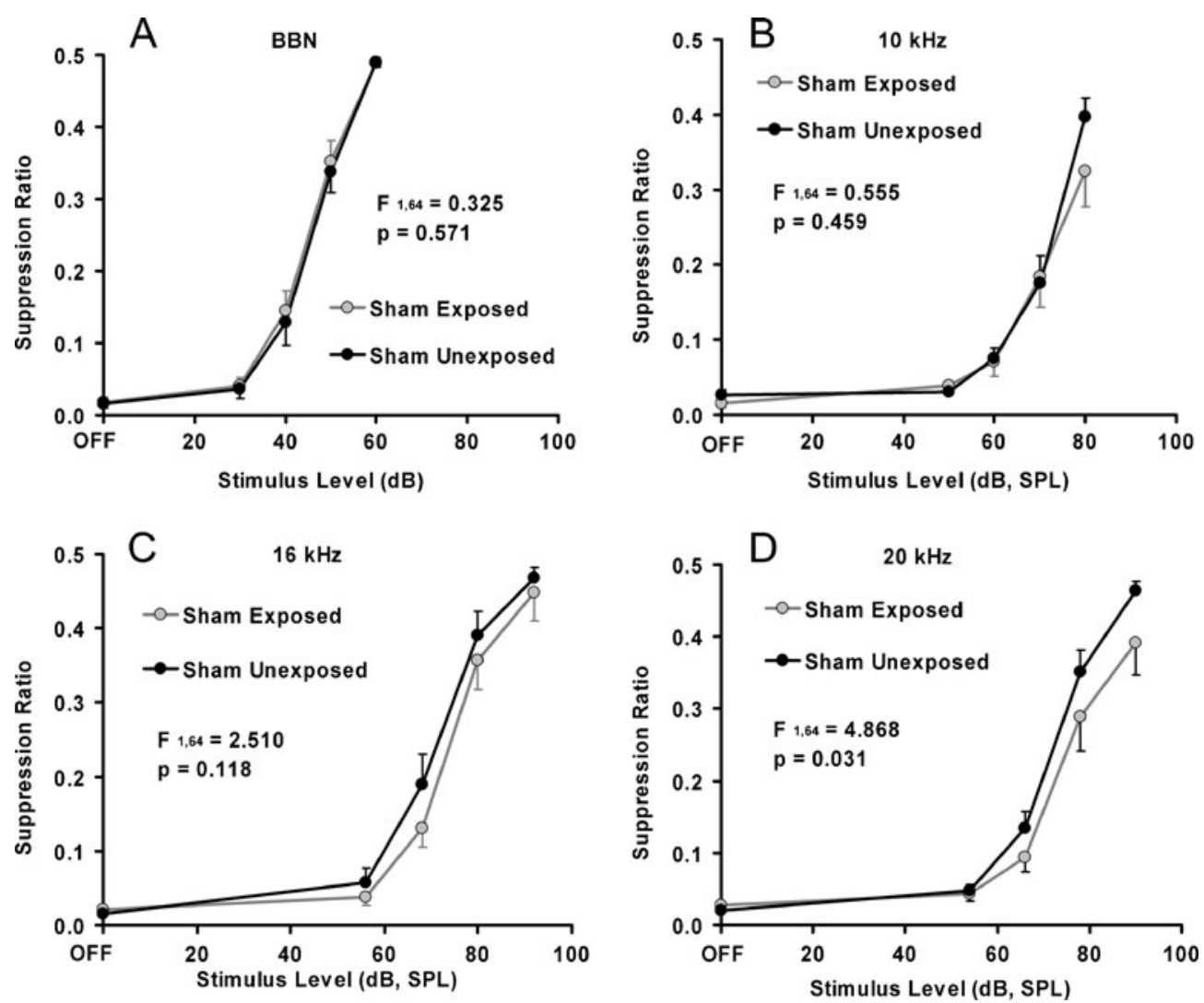

FIG. 3. A-D Evidence of tinnitus in DCN-intact rats. The discrimination functions of exposed and unexposed sham-lesioned (DCN intact) groups are compared across all test conditions. Group composition is summarized in Table 1. The statistical summary in each panel compares group performance across stimulus levels above speaker-off (mixed ANOVA with stimulus level as the repeated measure). The significant downshift (excess suppression) of the exposed group at $20 \mathrm{kHz}$ (D) indicates the presence of tinnitus. Error bars show the standard error of the mean.

high-level sound exposure and did not show evidence of tinnitus.

\section{Tinnitus: unilateral lesions}

Unilateral lesion results differed from bilateral lesion results. Neither ipsilateral nor contralateral lesions significantly protected the rats from unilateral soundinduced chronic tinnitus. Post-lesion psychophysical performance of both unilateral groups is compared to that of exposed DCN-intact rats in Figure 5. Unequal group numbers prevented ANOVA analysis, but multiple $t$ test comparisons showed no significant difference between the performance of exposed DCN-intact rats with tinnitus, and either of the exposed unilaterally lesioned groups ( $p=0.719$ to 0.894$)$. All three groups had similar performance, including the function downshift at $20 \mathrm{kHz}$ (Fig. 5D) indicative of tinnitus.

\section{Hearing thresholds}

Immediately after high-level sound exposure, ABR thresholds were elevated 30 to $50 \mathrm{~dB}$ in the exposed ear (Fig. 6, top panel, right). Unexposed ear thresholds for exposed animals were equivalent and within normal range (Fig. 6, top panel, left). At the conclusion of psychophysical testing, exposed ear thresholds were equivalently elevated by approximately $40 \mathrm{~dB}$ in all groups (Fig. 6, center panel, right; $p=0.763$ to 0.225 ), while unexposed ear thresholds were within normal range (Fig. 6, center panel, left; $p=$ 0.763 to 0.449$)$. Hearing thresholds for unexposed rats remained normal and were similar for all groups, lesioned as well as sham-lesioned (Fig. 6, bottom panel; $p=0.986$ to 0.392 ). To summarize, immediately after exposure, all groups displayed equivalent exposed ear threshold elevations and had persistent 40-dB elevations throughout testing. Unexposed animals, and the unexposed ears of exposed animals, displayed normal thresholds throughout.

\section{DISCUSSION}

Bilateral pre-exposure DCN lesions, unlike post-exposure lesions (Brozoski and Bauer 2005), prevented 

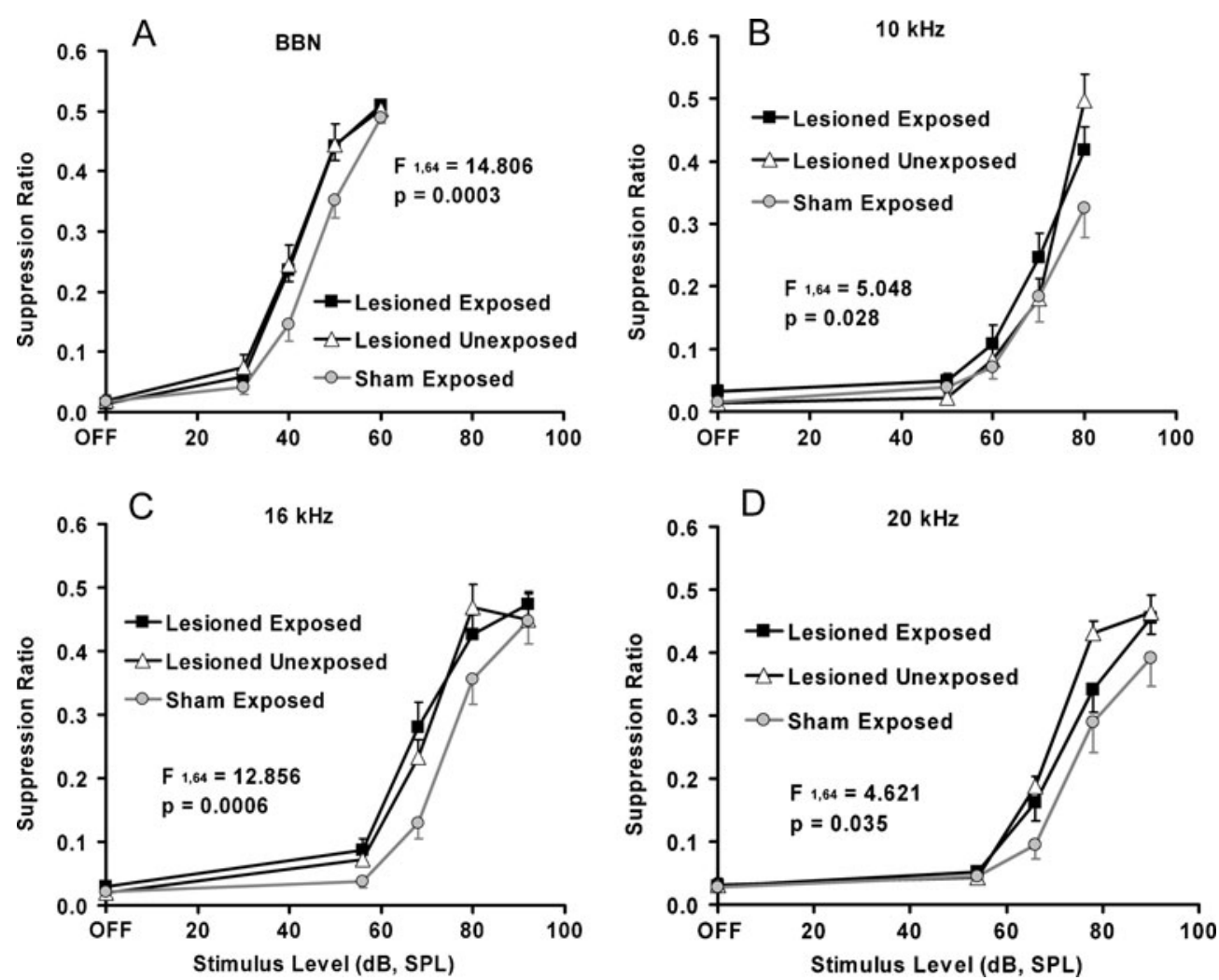

FIG. 4. A-D The protective effect of bilateral DCN lesions. Discrimination functions of the exposed bilaterally lesioned, unexposed bilaterally lesioned, and exposed sham-lesioned groups. Group composition is summarized in Table 1. The statistical comparison in each panel is between the exposed lesioned and exposed sham-lesion (DCN-intact) group, at stimulus levels above speaker-off (mixed ANOVA with stimulus level as the repeated measure). The exposed bilaterally lesioned group was significantly different than the exposed-intact group in all test conditions, but was not statistically different than the unexposed bilaterally lesioned group (see text for statistics). Error bars show the standard error of the mean.

acoustic trauma-induced chronic tinnitus in rats. In contrast, unilateral pre-exposure lesions did not prevent the development of tinnitus.

\section{Hearing loss and tinnitus}

The persistent unilateral threshold elevations, obtained in the present study, stand in contrast to previous studies using similar exposures. In those studies, the rats typically showed negligible to small $(10 \mathrm{~dB})$ permanent threshold shifts (Brozoski and Bauer 2005, 2007b). The approximate 40-dB elevation in the present study may have been the result of the isoflurane anesthesia used during exposure. Previous studies used a ketamine-xylazine mixture. Anesthetic used during acoustic exposure has been shown to modulate the level of post-exposure pathology. Isoflurane has complex central effects, inhibiting both excitatory glutamatergic and inhibitory GABAergic systems (Franks 2008). Most studies report reduced amplitude of evoked activity (Ferber-Viart et al. 1998; Heneghan et al. 1987; Santarelli et al. 2003), reduced steady-state-driven activity (Cheung et al. 2001; Plourde et al. 1998), and otoprotection from high- level exposure (Chung et al. 2007; Kim et al. 2005). The permanent ipsilateral threshold elevation in the present study remains to be explained. However, both lesioned and nonlesioned (exposed) rats had similar threshold elevations (Fig. 6, top panel, right ear), while DCN lesions alone did not affect thresholds (Fig. 6, top panel).

Could the unilateral hearing loss have been responsible for effects interpreted as tinnitus? Previous research has shown that unilateral threshold elevation, such as that produced by an ear plug, did not affect performance (Bauer and Brozoski 2001). Since psychophysical testing was done in a free sound field, information from both ears guides performance and unilateral threshold elevation does not significantly impact performance. In the present results, it is also clear that there was no systematic difference between exposed and unexposed performance (Fig. 4), as would be expected if there was an impact of hearing loss.

\section{DCN lesions and high-level sound exposure}

Could the tinnitus protective effect of bilateral DCN lesions be explained by a loss of ipsilateral 

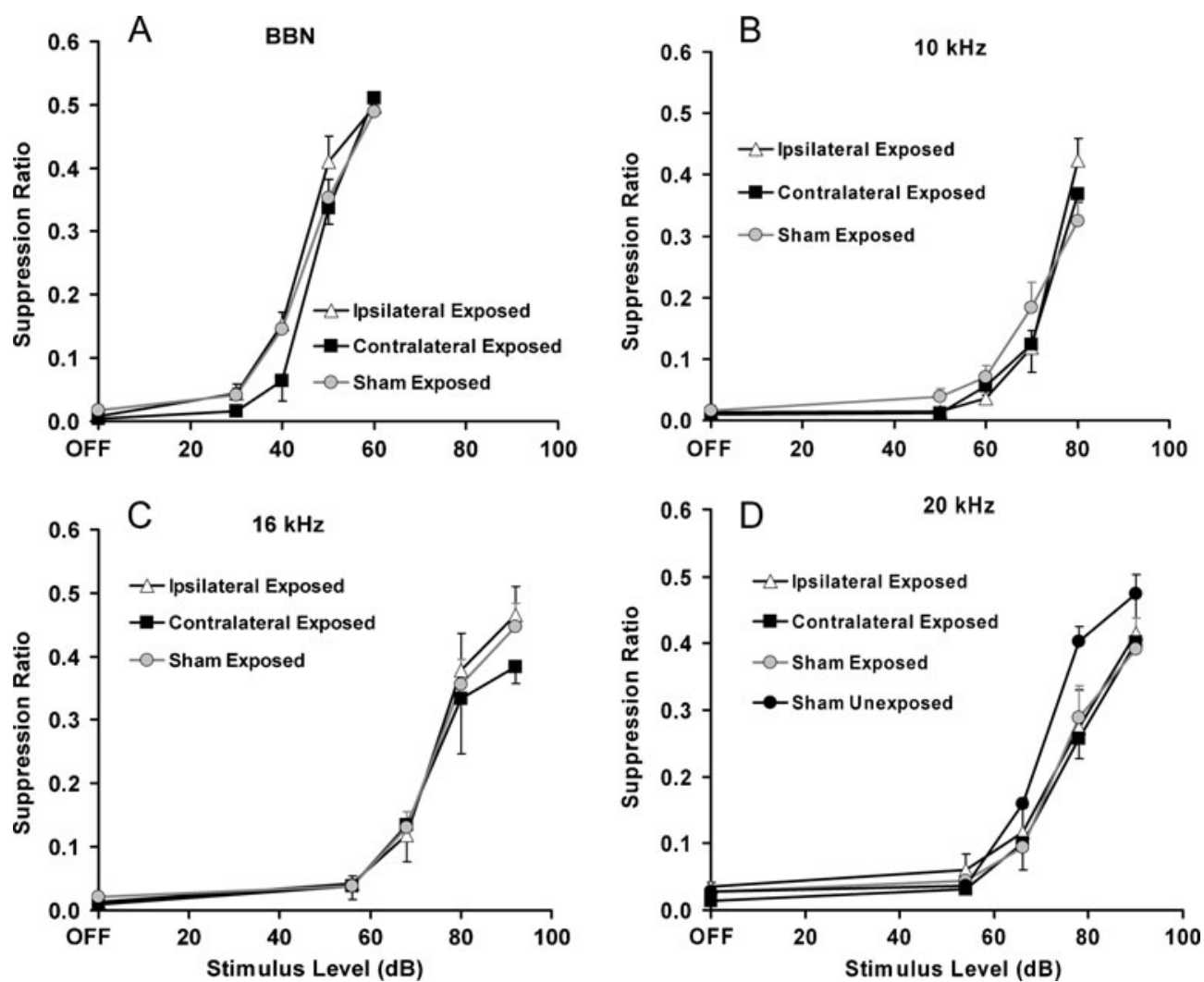

FIG. 5. A-D Unilateral DCN lesions did not confer a tinnitus protective effect. Compared are the discrimination functions of the exposed ipsilaterally lesioned, exposed contralaterally lesioned, and exposed sham-lesioned. D Includes the discrimination function of sham-lesioned traumatic sound-unexposed (i.e., no tinnitus) rats for comparison. Group composition is summarized in Table 1. All of the exposed groups showed significant evidence of tinnitus at $20 \mathrm{kHz}$ and were not statistically different from one another. Error bars show the standard error of the mean.

sensitivity, i.e., the lesion attenuated exposure sound loudness? If decreased stimulus drive during exposure was the tinnitus protective mechanism, then ipsilateral DCN lesions should have afforded as much protection as bilateral lesions. That was not the case. Furthermore, immediate post-exposure ABR threshold elevations for ipsilateral and bilateral lesions were not significantly different from one another or from DCN-intact rats (ipsilateral lesioned, exposed ear average $70.5 \mathrm{~dB}$, SPL vs. sham-lesioned, exposed ear average $73.9 \mathrm{~dB}, p=$ 0.608 ; vs. bilaterally lesioned exposed ear average $72.1 \mathrm{~dB}, p=0.702)$. Since ipsilateral ABR thresholds were equivalently elevated in all exposed groups, but tinnitus was only absent from the bilaterally lesioned group, protection from tinnitus would appear to derive from something other than afferent impairment during exposure.

\section{Potential mechanisms of ablation protection}

The primary hypothesis was that chronic tinnitus emerges as an overcompensation for long-term altered input produced by acoustic trauma. There is ample evidence that abnormally elevated activity appears in the cochlear nucleus following sound trauma. Most evidence points to the DCN (Kaltenbach 2006), although other areas of the cochlear nucleus may be involved (Shore and Zhou 2006). The compensatory mechanism may be a downregulation of GABA (Brozoski et al. 2007a, b) and glycine (Wang et al. 2009) or an upregulation of excitation (Bledsoe et al. 2009). An equally plausible compensatory mechanism may be the upregulation, and/or reorganization of excitatory input to the cochlear nucleus from nonauditory sources (Shore et al. 2008; Zeng et al. 2009). These mechanisms are not mutually exclusive, and in the DCN, the net effect would be manifested as inappropriately elevated spontaneous activity (Brozoski et al. 2002; Kaltenbach and McCaslin 1996; Kaltenbach and Heffner 1999). The elevated activity may persist because the DCN contains plastic circuits that facilitate adaptation to acoustic environments (Nelken and Young 1996; Tzounopoulos 2008; Young and Sachs 2008). The question posed in the present research was whether altered DCN output serves as a chronic tinnitus source, or alternatively, does it serve as a 

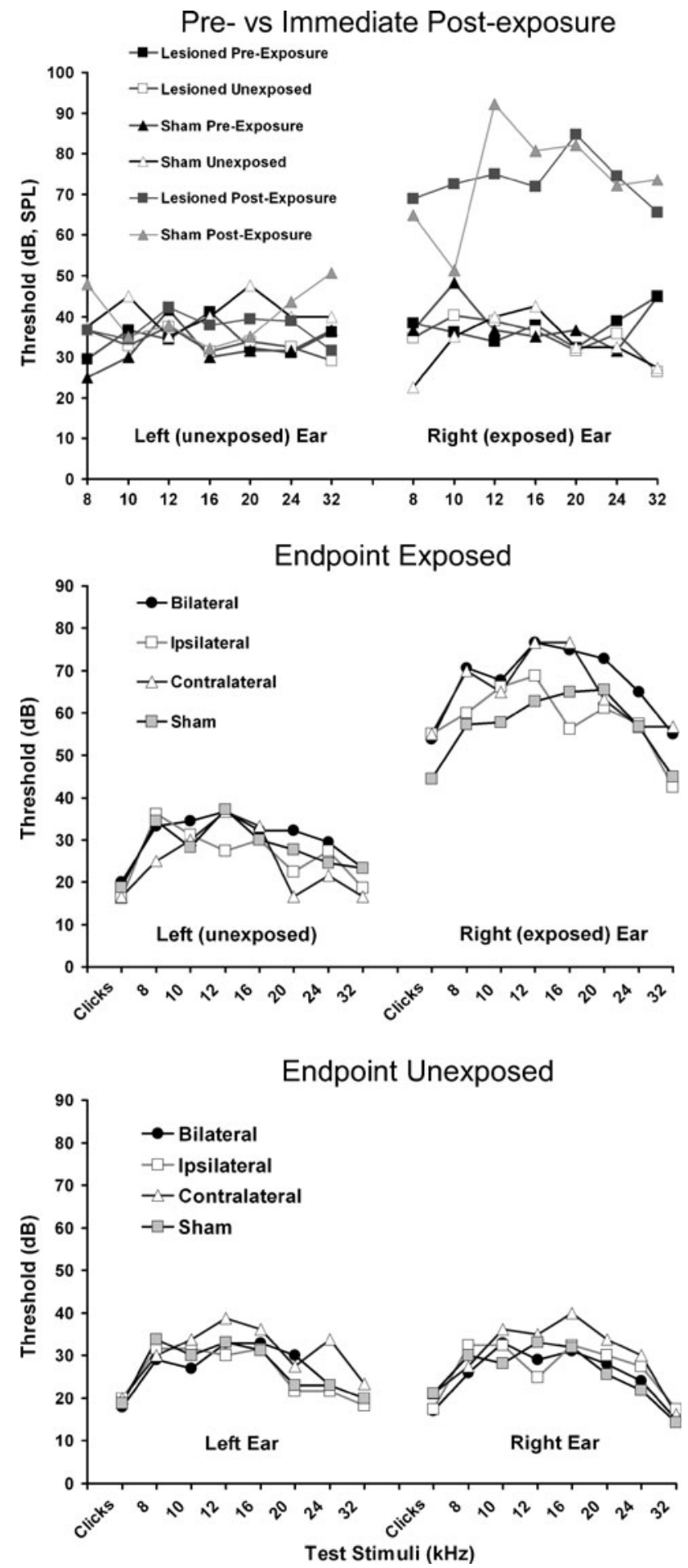

FIG. 6. Acoustic brainstem response (ABR) hearing thresholds. Top panel: Immediately before and after unilateral noise exposure. Both ablated and sham-lesioned traumatic sound-exposed groups showed a 40-50-dB threshold elevation in their exposed ears, but no elevation in their unexposed ears. Center panel: ABR thresholds at the conclusion of psychophysical testing for all exposed animals. An approximate 40-dB permanent threshold elevation was evident in their exposed ears and normal thresholds in their unexposed ears. There were no significant differences between animals with and without DCN lesions. Bottom panel: $\mathrm{ABR}$ thresholds at the conclusion of psychophysical testing for all unexposed animals. Thresholds were normal with no significant differences between animals with and without DCN lesions. trigger for alterations elsewhere in the system that then serve as the chronic source? The results suggest that the DCN serves more as a necessary trigger, than a necessary chronic generator. Lesions were made to disrupt the rostral output of the DCN before acoustic trauma. Some lesions were also extensive enough to disrupt intrinsic DCN activity, i.e., signal processing and connections to the ventral cochlear nucleus (Cant and Benson 2003). Whereas a previous study showed that bilateral (or unilateral) lesions months after exposure failed to reduce tinnitus (Brozoski and Bauer 2005), in the present experiment, bilateral lesions, prior to exposure, prevented the emergence of tinnitus. The present results therefore support the hypothesis that the necessary role of the DCN in chronic tinnitus is to trigger changes in other areas that then serve as the chronic tinnitus signal source. How long DCN output must remain elevated, in order to effectively trigger tinnitus, remains to be answered.

\section{Alternatives to the DCN trigger hypothesis}

It is possible that the DCN does not serve as a direct trigger for tinnitus, or that the trigger mechanism is unrelated to increased DCN spontaneous activity. During high-level sound exposure, acutely driven activity in the DCN, as opposed to increased spontaneous activity, could be responsible for triggering critical downstream changes that serve as tinnitus generators. Recently, it has been shown that spontaneous auditory nerve activity may be necessary for increased spontaneous activity in the inferior colliculus following acoustic trauma (Mulders and Robertson 2009). It was hypothesized that auditory nerve activity may enable the expression of abnormal activity in a traumatized brainstem when intrinsic inhibition has been compromised. Evidence to the contrary would be that, at least in some individuals, tinnitus persists and may be exacerbated after total eighth nerve transection (Berliner et al. 1992).

\section{Bilateral DCN lesions and discrimination function upshift}

Unexpectedly, bilateral DCN lesions produced an upshift in discrimination functions in all stimulus conditions (Fig. 4). The upshift may be interpreted as a nonspecific gain in supra-threshold loudness. Note that hearing threshold was not affected by bilateral DCN lesions (Fig. 6, top panel, unfilled square data points). Several lines of evidence suggest that the cerebellar-like circuitry of the DCN facilitates adaptive signal processing in dynamic acoustic environments (Oertel and Young 2004). One such process 
may be the output of a "negative image" of excitatory input. A negative image could serve an automatic gain control function, attenuating the influence of already detected signals (Bell et al. 2008). Bilateral DCN lesions would be expected to affect more than one aspect of DCN output. The upshifted discrimination functions of bilaterally lesioned DCN animals suggest an enhanced loudness growth function, perhaps the result of decreased feedforward inhibition. Another possibility is that loss of the tuberculoventral tract to the VCN releases that structure from feedback inhibition. Although this appears to be at odds with tinnitus protection, hyperacusis and tinnitus are different phenomena. It may be that elevated postexposure spontaneous activity (i.e., system noise) in the DCN was attenuated by lesions, while at the same time stimulus-driven output (i.e., signal), perhaps mediated by an intact ventral cochlear nucleus, was enhanced. These dual effects will have to be elucidated in future experiments.

\section{Bilateral vs. unilateral lesion effectiveness}

Why should bilateral but not ipsilateral DCN lesions attenuate tinnitus? Bilateral brainstem connections between the DCN are well known (Young and Brownell 1976). The present study suggests that downregulation of inhibition, or upregulation of excitation, following unilateral auditory insult occurs bilaterally in the brainstem. Bilateral elevation of DCN activity in unilaterally exposed chinchillas with tinnitus has been reported (Brozoski et al. 2002). Bilateral DCN interactions in acoustic processing are well documented. Contralateral acoustic input inhibits unit response in the DCN (Davis 2005; Ingham et al. 2006; Joris and Smith 1998; Young and Brownell 1976), as does electrical stimulation of the contralateral auditory nerve, indicated by electrophysiological data (Babalian et al. 1999) and c-Fos expression (Nakamura et al. 2005). Contralateral excitatory glutamatergic connections between the cochlear nuclei have been documented and may be more prevalent than inhibitory connections in the granule cell layer (Zhou et al. 2010). Contralateral hearing loss has been reported to strengthen contralateral excitatory inputs to the $\mathrm{CN}$ (Bledsoe et al. 2009). In sum, diverse studies suggest that bilateral and unilateral DCN lesions would have significantly different effects. The present results indicate that tinnitus pathology likely involves multiple structures that act in concert to produce the sensation of ringing in the ears. Further research will be necessary to understand the apparent obligatory bilateral involvement of the DCN as a tinnitus trigger.

\section{ACKNOWLEDGMENTS}

This study was supported by the National Institute on Deafness and Other Communication Disorders, \#1R01DC009669-01. We thank Lauren Ely and Kelsey Haines for their technical support.

\section{Open Access}

This article is distributed under the terms of the Creative Commons Attribution Noncommercial License which permits any noncommercial use, distribution, and reproduction in any medium, provided the original author(s) and source are credited.

\section{REFERENCES}

Babalian AL, Ryugo DK, Vischer MW, Rouiller EM (1999) Inhibitory synaptic interactions between cochlear nuclei: evidence from an in vitro whole brain study. Neuroreport 10:1913-1917

Backoff PM, Shadduck Palombi P, Caspary DM (1999) Gammaaminobutyric acidergic and glycinergic inputs shape coding of amplitude modulation in the chinchilla cochlear nucleus. Hear Res 134:77-88

Bauer CA, Brozoski TJ (2001) Assessing tinnitus and prospective tinnitus therapeutics using a psychophysical animal model. J Assoc Res Otolaryngol 2:54-64

Bauer CA, Brozoski TJ, Rojas R, Boley J, Wyder M (1999) Behavioral model of chronic tinnitus in rats. Otolaryngol Head Neck Surg 121:457-462

Bell CC, Han V, Sawtell NB (2008) Cerebellum-like structures and their implications for cerebellar function. Annu Rev Neurosci 31:1-24

Berliner Ki, Shelton C, Hitselberger WE, Luxford WM (1992) Acoustic tumors: effect of surgical removal on tinnitus. Am J Otol 13:13-17

Bledsoe SC Jr, Koehler S, Tucci DL, Zhou J, Le Prell C, Shore SE (2009) Ventral cochlear nucleus responses to contralateral sound are mediated by commissural and olivocochlear pathways. J Neurophysiol 102:886-900

Brozoski TJ, BAuer CA (2005) The effect of dorsal cochlear nucleus ablation on tinnitus in rats. Hear Res 206:227-236

Brozoski TJ, BAuEr CA (2008) Learning about tinnitus from an animal model. Semin Hear 29:242-258

Brozoski TJ, Bauer CA, Caspary DM (2002) Elevated fusiform cell activity in the dorsal cochlear nucleus of chinchillas with psychophysical evidence of tinnitus. J Neurosci 22:2383-2390

Brozoski TJ, Ciobanu L, Bauer CA (2007A) Central neural activity in rats with tinnitus evaluated with manganese-enhanced magnetic resonance imaging (MEMRI). Hear Res 228:168-179

Brozoski TJ, SpIres TJ, BAuer CA (2007в) Vigabatrin, a GABA transaminase inhibitor, reversibly eliminates tinnitus in an animal model. J Assoc Res Otolaryngol 8:105-118

Cant NB, Benson CG (2003) Parallel auditory pathways: projection patterns of the different neuronal populations in the dorsal and ventral cochlear nuclei. Brain Res Bull 60:457-474

Caspary DM, Pazara KE, Kossl M, Faingold CL (1987) Strychnine alters the fusiform cell output from the dorsal cochlear nucleus. Brain Res 417:273-282 
Cheung SW, Nagarajan SS, Bedenbaugh PH, Schreiner CE, Wang X, Wong A (2001) Auditory cortical neuron response differences under isoflurane versus pentobarbital anesthesia. Hear Res 156:115-127

Chung JW, Ahn JH, Kim JY, Lee HJ, Kang HH, Lee YK, Kim JU, KoO SW (2007) The effect of isoflurane, halothane and pentobarbital on noise-induced hearing loss in mice. Anesth Analg 104:14041408 , table of contents

DAvis KA (2005) Contralateral effects and binaural interactions in dorsal cochlear nucleus. J Assoc Res Otolaryngol 6:280-296

Dehmel S, Cu YL, Shore SE (2008) Cross-modal interactions of auditory and somatic inputs in the brainstem and midbrain and their imbalance in tinnitus and deafness. Am J Audiol 17:S193-S209

Eggermont JJ, Roberts LE (2004) The neuroscience of tinnitus. Trends Neurosci 27:676-682

Ferber-Viart C, Preckel MP, Dubreuil C, Banssillon V, Duclaux R (1998) Effect of anesthesia on transient evoked otoacoustic emissions in humans: a comparison between propofol and isoflurane. Hear Res 121:53-61

Franks NP (2008) General anaesthesia: from molecular targets to neuronal pathways of sleep and arousal. Nat Rev Neurosci 9:370-386

Guitton MJ, Caston J, Ruel J, Johnson RM, Pujol R, Puel JL (2003) Salicylate induces tinnitus through activation of cochlear NMDA receptors. J Neurosci 23:3944-3952

Heffner HE, Harrington IA (2002) Tinnitus in hamsters following exposure to intense sound. Hear Res 170:83-95

Heneghan CP, Thornton C, Navaratnarajah M, Jones JG (1987) Effect of isoflurane on the auditory evoked response in man. $\mathrm{Br}$ J Anaesth 59:277-282

InGHAM NJ, BleECK S, Winter IM (2006) Contralateral inhibitory and excitatory frequency response maps in the mammalian cochlear nucleus. Eur J Neurosci 24:2515-2529

Iто MI (1984) The cerebellum and neural control. Raven, New York

Jastreboff PJ, Brennan JF, Coleman JK, Sasaki CT (1988) Phantom auditory sensation in rats: an animal model for tinnitus. Behav Neurosci 102:811-822

Joris PX, Sмгтн PH (1998) Temporal and binaural properties in dorsal cochlear nucleus and its output tract. J Neurosci 18:10157-10170

Kaltenbach JA (2006) The dorsal cochlear nucleus as a participant in the auditory, attentional and emotional components of tinnitus. Hear Res 216-217:224-234

Kaltenbach JA, McCaslin DL (1996) Increases in spontaneous activity in the dorsal cochlear nucleus following exposure to high intensity sound: a possible neural correlate of tinnitus. Auditory Neurosci 3:57-78

Kaltenbach JA, Heffner HE (1999) Spontaneous activity in the dorsal cochlear nucleus of hamsters tested behaviorally for tinnitus. (Abstract). Association for Research in Otolaryngology Midwinter Research Meeting, St. Petersburg Beach

Kaltenbach JA, Afman CE (2000) Hyperactivity in the dorsal cochlear nucleus after intense sound exposure and its resemblance to tone-evoked activity: a physiological model for tinnitus. Hear Res 140:165-172

Kaltenbach JA, Zhang J, Afman CE (2000) Plasticity of spontaneous neural activity in the dorsal cochlear nucleus after intense sound exposure. Hear Res 147:282-292
Kim JU, Lee HJ, Kang HH, Shin JW, Ku SW, Ahn JH, Kim YJ, Chung JW (2005) Protective effect of isoflurane anesthesia on noise-induced hearing loss in mice. Laryngoscope 115:1996-1999

Lobarinas E, Sun W, Cushing R, SAlvi R (2004) A novel behavioral paradigm for assessing tinnitus using schedule-induced polydipsia avoidance conditioning (SIP-AC). Hear Res 190:109-114

Mulders WH, RoberTson D (2009) Hyperactivity in the auditory midbrain after acoustic trauma: dependence on cochlear activity. Neuroscience 164:733-746

Nakamura M, Rosahl SK, Alkahlout E, Walter GF, Sami MM (2005) Electrical stimulation of the cochlear nerve in rats: analysis of c-Fos expression in auditory brainstem nuclei. Brain Res 1031:39-55

Nelken I, Young ED (1996) Why do cats need a dorsal cochlear nucleus? J Basic Clin Physiol Pharmacol 7:199-220

Oertel D, Young ED (2004) What's a cerebellar circuit doing in the auditory system? Trends Neurosci 27:104-110

Plourde G, Villemure C, Fiset P, Bonhomme V, Backman SB (1998) Effect of isoflurane on the auditory steady-state response and on consciousness in human volunteers. Anesthesiology 89:844-851

Reiss LA, Bandyopadhyay S, Young ED (2007) Effects of stimulus spectral contrast on receptive fields of dorsal cochlear nucleus neurons. J Neurophysiol 98:2133-2143

Ruttiger L, Ciuffani J, Zenner HP, Knipper M (2003) A behavioral paradigm to judge acute sodium salicylate-induced sound experience in rats: a new approach for an animal model on tinnitus. Hear Res 180:39-50

Santarelli R, Arslan E, Carraro L, Conti G, Capello M, Plourde G (2003) Effects of isoflurane on the auditory brainstem responses and middle latency responses of rats. Acta Otolaryngol 123:176181

Shore SE, Zhou J (2006) Somatosensory influence on the cochlear nucleus and beyond. Hear Res 216-217:90-99

Shore SE, Koehler S, Oldakowski M, Hughes LF, Syed S (2008) Dorsal cochlear nucleus responses to somatosensory stimulation are enhanced after noise-induced hearing loss. Eur J Neurosci 27:155-168

Tzounopoulos $\mathrm{T}$ (2008) Mechanisms of synaptic plasticity in the dorsal cochlear nucleus: plasticity-induced changes that could underlie tinnitus. Am J Audiol 17:S170-S175

Wang H, Brozoski TJ, Turner JG, Ling L, Parrish JL, Hughes LF, Caspary DM (2009) Plasticity at glycinergic synapses in dorsal cochlear nucleus of rats with behavioral evidence of tinnitus. Neuroscience 164:747-759

Young ED, Brownell WE (1976) Responses to tones and noise of single cells in dorsal cochlear nucleus of unanesthetized cats. J Neurophysiol 39:282-300

Young ED, SACHS MB (2008) Auditory nerve inputs to cochlear nucleus neurons studied with cross-correlation. Neuroscience 154:127-138

Zeng C, Nannapaneni N, Zhou J, Hughes LF, Shore S (2009) Cochlear damage changes the distribution of vesicular glutamate transporters associated with auditory and nonauditory inputs to the cochlear nucleus. J Neurosci 29:4210-4217

Zhou J, Zeng C, Cui Y, Shore S (2010) Vesicular glutamate transporter 2 is associated with the cochlear nucleus commissural pathway. J Assoc Res Otolaryngol 11:675-687 